\title{
Difficult structures of organic molecules and how to solve them
}

\author{
Marcus Müller, Dubravka Sisak Jung
}

DECTRIS Ltd., Baden-Daettwil, Switzerland

Marcus.mueller@dectris.com

\begin{abstract}
$\mathrm{Ab}$ initio structure determination from Powder X-ray Diffraction (PXRD) data is continuously demonstrating its merit thanks to advances in modern phasing algorithms, computing power and X-ray instrumentation. As the technique has long passed the question "can it be done", there is another question to answer: "how far can it be pushed". In this respect, this presentation has two aims: (i) to show methodologies that allow for solving of difficult structures of organic molecules and (ii) to highlight the level of accuracy that can be obtained from PXRD data.
\end{abstract}

This presentation is focused on structure determination of crystals that feature large molecules, disorder, or radiation-induced changes. The first part of the presentation outlines a phasing methodology that can result in an interpretable structural model. The methodology relies on the phasing process in the charge-flipping program [2] Superflip [3] by introducing a partial or incorrect structure obtained by a direct-space algorithm FOX [4]. The second part of the presentation will address structure completion and refinement, and highlight examples of how high quality data can be used for restraint-free Rietveld refinement, modeling disorder from difference Fourier map, and for obtaining insights in bond order disambiguation.

While the majority of shown examples rely on PXRD data collected at synchrotron sources, the potential of data collected in a laboratory diffractometer will also be discussed.

1. Šššak Jung, D. et al. (2014). J. Appl. Cryst. 47, 1569-74

2. Oszlanyi, G., Sütő, A. (2004). Acta Cryst. A60, 134-141

3. Palatinus, L., Chapuis, G. (2007). J. Appl. Cryst. 40, 786-790

4. Favre-Nicolin, V., Černý, R. (2004). Z. Kristallogr. 219(12), 847-856.

Keywords: Charge flipping, radiation sensitivity, structure determination 